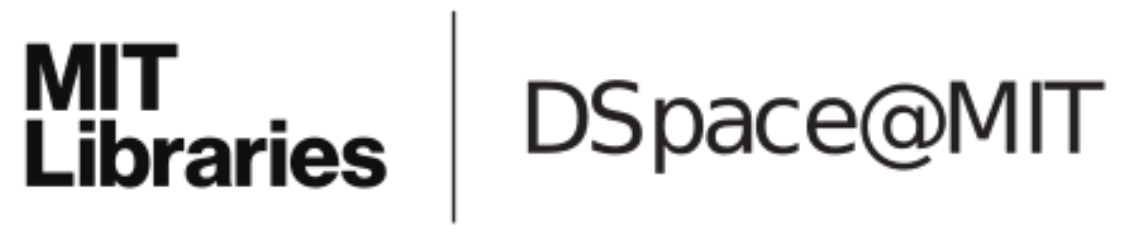

\author{
MIT Open Access Articles
}

A low order model for vertical axis wind turbines

The MIT Faculty has made this article openly available. Please share how this access benefits you. Your story matters.

Citation: Asher, Isaac M., Mark Drela and Jaime Peraire. "A Low Order Model for Vertical Axis Wind Turbines." in Proceedings of the 28th AIAA Applied Aerodynamics Conference 28 June-1 July 2010, Chicago, Illinois.

Publisher: American Institute of Aeronautics and Astronautics

Persistent URL: http://hdl.handle.net/1721.1/59430

Version: Author's final manuscript: final author's manuscript post peer review, without publisher's formatting or copy editing

Terms of use: Attribution-Noncommercial-Share Alike 3.0 Unported 


\title{
A Low Order Model for Vertical Axis Wind Turbines
}

\author{
Isaac M. Asher*, Mark Drela ${ }^{\dagger}$ and Jaime Peraire ${ }^{\ddagger}$ \\ Massachusetts Institute of Technology, Cambridge, MA 02139, U.S.A.
}

\begin{abstract}
A new computational model for initial sizing and performance prediction of vertical axis wind turbines is presented. The model uses a 2D hybrid dynamic vortex and blade element momentum approach. Each airfoil is modeled as a single vortex of time varying strength with an analytical model for the influence of the shed vorticity. The vortex strengths are calculated by imposing a flow tangency condition at the three-quarter chord location on each airfoil, modified in the case of stall. The total blade forces and the momentum-based streamtube deceleration are then obtained using pre-computed $c_{d}$ and $c_{m}$ 2D blade profile characteristics. Model fidelity is improved over previous models because flow curvature, dynamic vortices, blade interactions, static stall, and streamtube changes are all taken into account. Fast convergence is obtained for a large range of solidity and tip speed ratio, which allows optimization of various parameters, including blade pitch angle variation.
\end{abstract}

\section{Nomenclature}

Letters

$A \quad$ frontal area, taken as $2 R$ (unit span).

$A_{0}, A_{1}$ pitch angle modulation coefficients.

$b \quad=2 / \pi$, wake vorticity calibration constant.

$c \quad$ blade airfoil chord.

$C(\Gamma)$ stall model leakage velocity.

$c_{d} \quad$ coefficient of drag.

$c_{l} \quad$ coefficient of lift computed using $\Gamma$.

$\Delta c_{l}=0.05$, stall onset parameter.

$c_{l \min , \max }$ stall thresholds.

$c_{m} \quad$ coefficient of moment.

$C_{P}=N_{b} \bar{P} / \frac{1}{2} \rho V_{\infty}^{3} A$, average power coefficient.

$C_{T}=N_{b} \bar{T} / \frac{1}{2} \rho V_{\infty}^{2} A$, average thrust coefficient.

Note that $C_{T}>0$ corresponds to windmilling.

$\vec{D} \quad=\frac{1}{2} \rho \vec{V}_{c / 4}^{2} c c_{d}$, drag force (along $\vec{V}_{c / 4}$ ).

$h \quad=\frac{1}{2}$, lift curve slope parameter.

$K_{s}=40$, stall 'leakage' velocity factor.

$K_{c_{d}} \quad$ drag coefficient factor.

$\vec{L}^{2}=\rho \Gamma\left(\vec{V}_{c / 4} \times-\hat{z}\right)+\rho c \dot{\Gamma}\left(\vec{V}_{c / 4} \times-\hat{z}\right) / V_{c / 4}$, lift force.

$\ell \quad$ stream-tube parameter.

$\vec{M} \quad=\frac{1}{2} \rho\left(\vec{V}_{c / 4}^{2} \times \hat{\eta}\right) c^{2} c_{m}$,

moment, positive counter clockwise.

$\vec{M}_{\text {tot }}=\vec{M}+\vec{r}_{c / 4} \times(\vec{L}+\vec{D})$,

moment due to $\vec{M}$, drag, and lift.

$N_{b} \quad$ number of blades.

$\vec{n} \quad$ unit vector normal to airfoil camber line at $\vec{r}_{\mathrm{cp}}$.
$P \quad=\vec{M}_{\text {tot }} \cdot \vec{\Omega}$, instantaneous power.

$R \quad$ radius of turbine (origin to leading edge of blade).

$\vec{r}_{c / 4} \quad$ quarter chord point.

$\vec{r}_{\mathrm{cp}} \quad$ control point, the three-quarter chord.

$\vec{r}_{\mathrm{le}} \quad$ vector from $x, y$ origin to leading edge of foil.

Re chord Reynolds number.

$T \quad$ thrust (component of $\vec{L}$ and $\vec{D}$ in $\hat{x}$-direction).

TSR $=\Omega R / V_{\infty}$, tip speed ratio.

$\vec{V}_{\infty} \quad$ external freestream velocity far upstream.

$\vec{V}_{\infty}^{\prime}(\vec{r})$ external freestream velocity evaluated at $\vec{r}$ using stream tube deceleration model.

$\vec{V}_{\text {rot }}=-\vec{\Omega} \times \vec{r}$ velocity due to rotation $\vec{\Omega}$,

$\vec{V}_{\mathrm{cp}} \quad$ relative velocity at the control point

$\vec{V}_{c / 4} \quad$ relative velocity at the quarter chord used for calculating aerodynamic forces, includes vortex-induced velocities.

$\vec{V}_{\Gamma} \quad=-\Gamma \vec{n} / \pi c$, velocity induced at $\vec{r}_{\mathrm{cp}}$ by the vortex at $\vec{r}_{c / 4}$.

$\vec{V}_{w} \quad=\left[-b \dot{\Gamma} / \vec{V}_{\mathrm{cp}} \cdot \hat{s}\right] \vec{n}$, wake-induced velocity.

Symbols

$\alpha_{0} \quad$ angle between $-\hat{\theta}$ and chord line $\hat{s}$, positive clockwise about the leading edge.

$\Gamma \quad$ strength of vortex located at $\vec{r}_{c / 4}$, positive clockwise.

$\theta \quad$ rotation angle of blade, measured counter-clockwise from $+y$-axis.

$\rho \quad$ density of air.

${ }^{*}$ Research Assistant, Dept. of Aeronautics and Astronautics, 77 Massachusetts Avenue Room 37-442, Cambridge, MA 02139, isaaca@alum.mit.edu, AIAA Student Member.

${ }^{\dagger}$ Terry J. Kohler Professor of Fluid Dynamics, Dept. of Aeronautics and Astronautics, AIAA Fellow.

${ }^{\ddagger}$ Professor, Dept. of Aeronautics and Astronautics, AIAA Associate Fellow. 


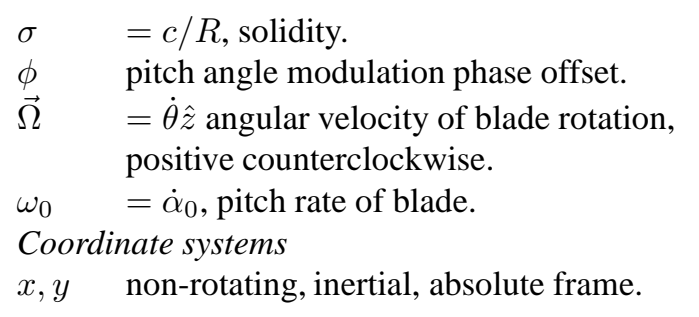
$r, \theta \quad$ frame centered at the leading edge of the airfoil with $\hat{r}$ away from the origin and $\hat{\theta}$ counterclockwise.
$s, t \quad$ frame centered at the leading edge of the airfoil with $\hat{s}$ along the chord line and $\hat{t}$ perpendicular.
$z \quad$ perpendicular to the plane (spanwise direction).

\section{Introduction}

Darrieus-type vertical axis wind turbines (VAWTs) have advantages in omni-directionality and structural simplicity over traditional horizontal axis wind turbines (HAWTs), but they are more complex aerodynamically. Current VAWT designs are in general not as efficient as HAWTs, and one contributing factor is a deficiency in design tools. Most simple, low order models cannot capture enough physics to reliably predict performance. More accurate models tend to be too slow and prevent fast design iterations. The tool developed in this project is computationally efficient and incorporates many of the salient features of the accurate tools, thus giving the designer the ability to search the design space effectively.

The model uses the Blade Element/Momentum method introduced by Templin ${ }^{1}$. The entire turbine is assumed to be a single actuator disc. The blade interactions captured by the more intricate multiple and double-multiple streamtube models of Strickland ${ }^{2}$ and Paraschivoiu ${ }^{3}$ are here captured with the airfoil vortices. Variations in the streamtube deceleration function form (constant, linear, and inverse tangent) had little effect on the resulting $C_{P}$, so it is assumed that the single actuator disc with linear deceleration is sufficiently accurate.

The airfoils are modeled by a vortex at the quarter chord of varying strength as Strickland ${ }^{4}$ did. The shed vorticity (due to varying vortex strength) is set to a $2 \mathrm{D}$ analytic approximation. The model does not track the shed vorticity, since its influence is generally small far from the airfoil. Strickland extended his vortex method to include static and dynamic stall and 'flow curvature'. Static stall and flow curvature are both included in this model.

The model is a hybrid between the Blade Element/Momentum and Vortex methods, and is accurate and fast enough to be wrapped in an optimization loop and give good results. One shortcoming is that solidity must not be allowed to grow too large during optimization, because there is little penalty in the model for very high solidities (blade interactions are approximate).

This model does not include 3D effects that are common to previous models, although wind shear and tip vortices could easily be included. At the moment, the model is useful for assessing the performance of straight-bladed turbines with high blade aspect ratios, moderate to low solidities, and moderate to high tip speed ratios.

\section{Assumptions}

We take the simple case of a 2D airfoil rotating in the plane at constant angular velocity $\Omega$ (see Fig. 1 for geometry setup). The freestream is equal to $\vec{V}_{\infty}$ far from the airfoil. Near the airfoil, the freestream decelerates linearly with the net deceleration determined by the average thrust produced by the airfoil ${ }^{*}$. The air flow around the airfoil is assumed to be incompressible and to have constant Reynolds and Mach numbers. The precise velocity and pressure distributions are approximated by replacing the airfoils with a discrete set of vortices. One main vortex is located at the quarter chords of the airfoils, and a secondary wake vortex is located at the trailing edges ${ }^{\S}$. The net induced velocity from the freestream, kinematics (rotation and pitch angle changes), and the main vortices of other airfoils (though not the wake vortices of the other airfoils) is used to calculate the forces on a given vortex. Losses (drag) and moments are captured with locally 2D airfoil profile drag characteristics ${ }^{\top}$. The chosen reference units are $R=1, \vec{V}_{\infty}=1 \hat{x}$, and $\rho=1$. We enforce a flow tangency condition and the thrust coefficient equation and solve for $\Gamma(\theta)$ and $C_{T}$.

\footnotetext{
${ }^{*}$ The distance over which the flow decelerates is set a-priori.

$\S$ The wake vorticity is proportional to the shed vorticity $\dot{\Gamma}$.

9 Taken at the nominal Reynolds number. Given the vortex strengths, we can compute $c_{l}$ and use $c_{d}\left(c_{l}\right)$ data. For $c_{l}$ beyond stall, a linear extrapolation is used. The same is done for the moment, except that $c_{m}$ is assumed zero beyond stall. 


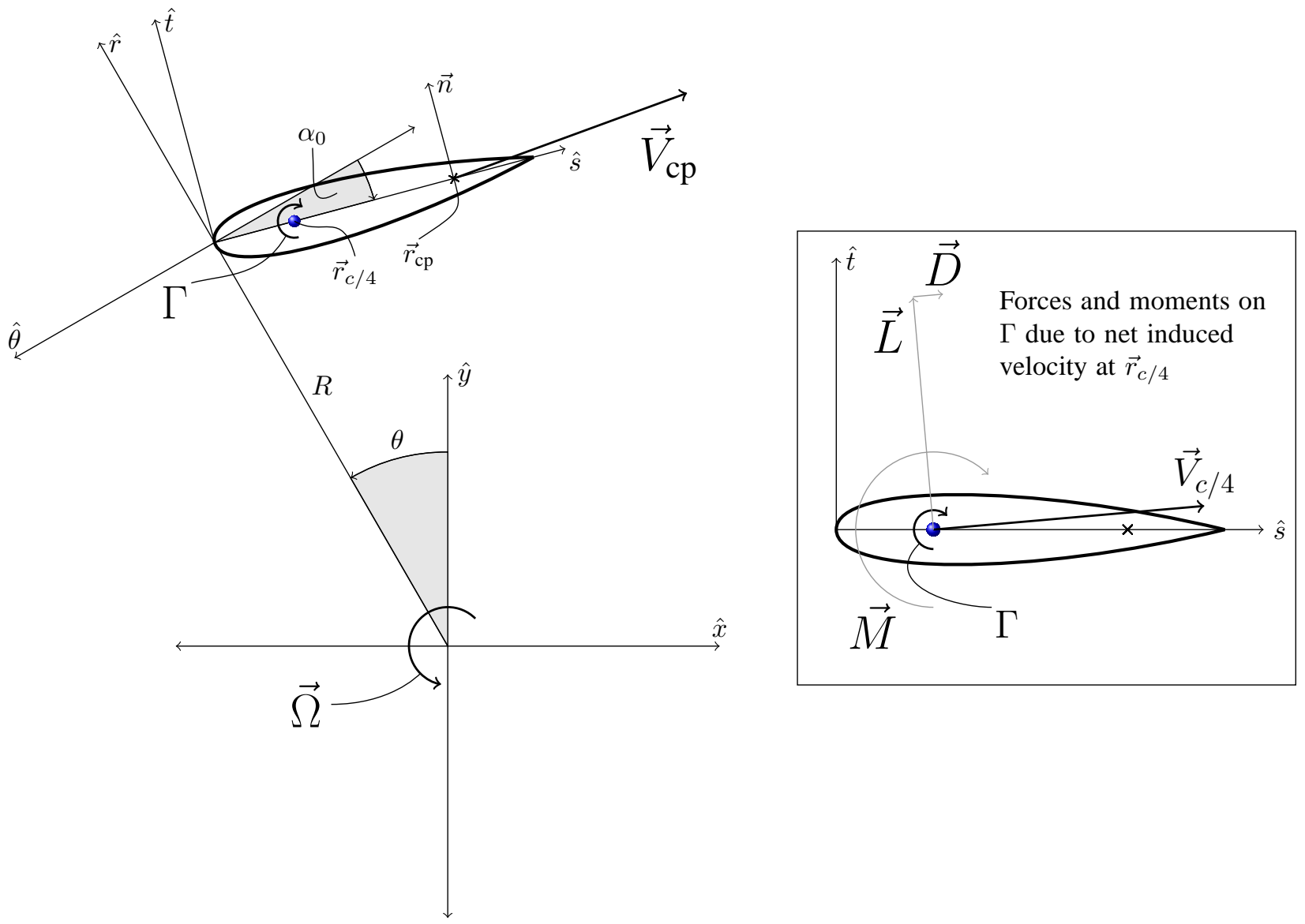

Figure 1. Turbine geometry and model setup

\section{Model Algorithm}

\section{A. Streamtube Deceleration}

For the streamtube model only, the turbine is assumed to be a single actuator disc, and $\vec{V}_{\infty}^{\prime}$ is calculated using the stream tube deceleration implied by $C_{T}$. A linear deceleration over a distance $2 \ell$ is assumed*,

$$
\vec{V}_{\infty}^{\prime}=\left\{\begin{array}{cl}
\vec{V}_{\infty}, & x<-\ell, \\
\vec{V}_{\infty}\left[1+\frac{\sqrt{1-C_{T}}-1}{2}\left(1+\frac{x}{\ell}\right)\right], & -\ell \leq x \leq \ell, \\
\vec{V}_{\infty}\left(\sqrt{1-C_{T}}\right), & x>\ell .
\end{array}\right.
$$

An example $\vec{V}_{\infty}^{\prime}$ distribution for $\ell=6$ and $C_{T}=0.8$ is show in Fig. 2.

\section{B. Blade-relative Velocities}

The various contributing velocities at $\vec{r}_{\mathrm{cp}}$ are calculated. The freestream velocity is calculated with the streamtube deceleration model. The kinematic velocity, or the blade-observed velocity due to its movement (varying $\theta$ and $\alpha_{0}$ ) is

$$
\vec{V}_{\mathrm{rot}}=-\left[\vec{\Omega} \times \vec{r}_{\mathrm{le}}+c \frac{3}{4} \dot{\hat{s}}\right] .
$$

${ }^{*} \ell$ is chosen a-priori

$$
3 \text { of } 9
$$


The total freestream velocity, which corresponds to the terms not multiplying $\Gamma$ in Eq. (2), is then

$$
\vec{V}_{\mathrm{cp}}=\vec{V}_{\infty}^{\prime}+\vec{V}_{\mathrm{rot}}=\vec{V}_{\infty}^{\prime}\left(\vec{r}_{\mathrm{cp}}\right)-\vec{\Omega} \times \vec{r}_{\mathrm{le}}-c \frac{3}{4} \dot{\hat{s}} .
$$

The velocity at $\vec{r}_{\mathrm{cp}}$ due to the main vortex (which becomes the term multiplying $\Gamma$ in Eq. (2)) is

$$
\vec{V}_{\Gamma}=-\Gamma \frac{\vec{n}}{\pi c} .
$$

Note that the direction is taken as $\vec{n}$, which is not strictly true if the airfoil has camber. However, it is important numerically to preserve the magnitude of $\vec{V}_{\Gamma}$.

The influence of the main vortices of the other airfoils are also calculated. The effect of the main vortex of foil $i$ on the control point for foil $j$ is

$$
\vec{V}_{\Gamma i, j}=\Gamma \frac{\vec{r}_{\mathrm{cp}, j}-\vec{r}_{c / 4, i}}{2 \pi\left|\vec{r}_{\mathrm{cp}, j}-\vec{r}_{c / 4, i}\right|^{2}} .
$$

The wake velocity is based on a calibrated approximation to the velocity induced by the trailing wake vortex sheet ${ }^{5}$,

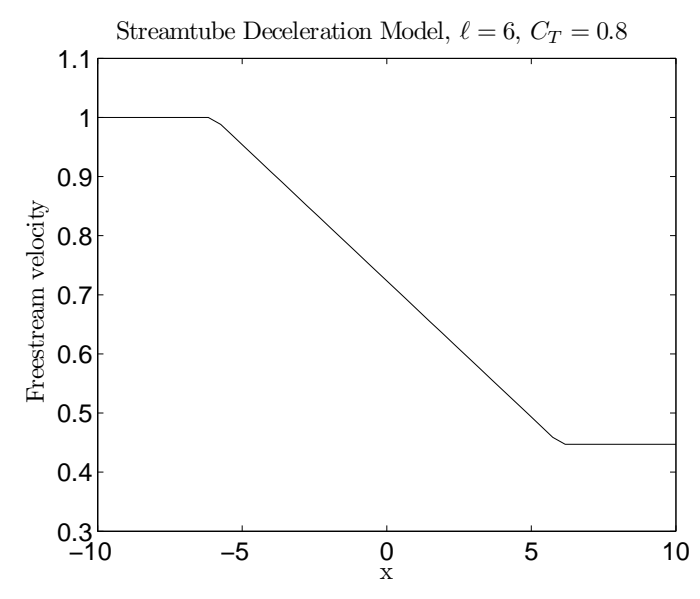

$$
\vec{V}_{w}=\frac{-b}{\vec{V}_{\mathrm{cp}}\left(\vec{r}_{\mathrm{cp}}\right) \cdot \hat{s}} \dot{\Gamma} \vec{n}
$$

Figure 2. Example of streamtube velocity distribution

This introduces a $\dot{\Gamma}$ term, which turns Eq. (2) into a rate equation for $\Gamma$.

\section{Flow Tangency and Stall Model}

The model assumes that the airfoil can be represented by a vortex at the quarter chord of strength $\Gamma(t)$. The standard practice is to compute a $\Gamma(\theta)$ to enforce flow tangency at a control point on the airfoil. That is, a $\Gamma$ is found such that

$$
\vec{V}\left(\vec{r}_{\mathrm{cp}}\right) \cdot \vec{n}=0 . \quad \text { no-stall }
$$

Here $\vec{V}$ includes contributions from the rotation $\left(\vec{V}_{\text {rot }}\right)$, the external flow $\left(\vec{V}_{\infty}^{\prime}\right)$, the vortex $\left(\vec{V}_{\Gamma}\right)$, and the wake $\left(\vec{V}_{w}\right)$. In this model, we add a simple stall model by setting the right hand side of Eq. (1) to some non-zero velocity 'leakage' at high $c_{l}$. The equation becomes

$$
\vec{V}\left(\vec{r}_{\mathrm{cp}}\right) \cdot \vec{n}=\frac{V_{\mathrm{cp}}}{4 \pi h} K_{s} \Delta c_{l} \log \frac{1+\exp \left[\left(c_{l}-c_{l \max }\right) / \Delta c_{l}\right]}{1+\exp \left[\left(c_{l \min }-c_{l}\right) / \Delta c_{l}\right]}, \quad \text { stall }
$$

where we take $h=1 / 2, K_{s}=40$, and $\Delta c_{l}=0.05 . c_{l \max }$ and $c_{l \min }$ are found manually. The leakage velocity is plotted in Fig. 3 versus $c_{l}$.

The control point is chosen to be the $\frac{3}{4}$-chord point ${ }^{\|}$to be consistent with thin airfoil theory. The normal vector is perpendicular to the airfoil camber line at the control point (at $x=\frac{3}{4}$ ).

Once a $\Gamma$ is found to satisfy Eq. (2), outputs such as lift, drag, moment, power out, and power coefficient may be computed. Lift is computed using the vortex strength, and drag and moment are computed using the lift and 2D airfoil section polars. Power coefficient is computed at each $\theta$ and integrated numerically to obtain $C_{P}$.

\footnotetext{
${ }^{\|}$Note that $\vec{r}_{\mathrm{cp}}$ here always lies along the chord line, rather than on the camber line or aligning with the local freestream. This is done to simplify the computation.
} 


\section{Circulation Evolution Equation}

Now Eq. (2) is of the form

$$
\left(-\frac{\vec{n}}{\pi} \Gamma+\vec{V}_{\mathrm{cp}}+\frac{-b}{\vec{V}_{\mathrm{cp}} \cdot \hat{s}} \dot{\Gamma} \vec{n}\right) \cdot \vec{n}=C(\Gamma),
$$

where $C$ is the right hand side that constitutes the stall model (which depends on $c_{l}$ and therefore $\Gamma$ ). Note that $C_{T}$ is an input (needed to calculate $\vec{V}_{\mathrm{cp}}$ ) and an output (based on forces generated), so both $\Gamma$ and $C_{T}$ must be computed iteratively. This is done by Newton iterations to drive the residual of the above equation to zero. In order to obtain an expression for $C_{T}$, we need to calculate the forces on the blades.

\section{E. Force and Moment Calculation}

Given a guess of $\Gamma$ and $C_{T}$, the velocity at any point in the resulting flow field can be found. Forces and moments are evaluated using the 'infinity' velocity at the vortex location

$$
\vec{V}_{c / 4}=\vec{V}\left(\vec{r}_{c / 4}\right)=\vec{V}_{\infty}^{\prime}\left(\vec{r}_{c / 4}\right)-\vec{\Omega} \times \vec{r}_{\mathrm{le}}-c \frac{1}{4} \dot{\hat{s}}-\frac{b \dot{\Gamma} \vec{n}}{\vec{V}_{\mathrm{cp}}\left(\vec{r}_{\mathrm{cp}}\right) \cdot \hat{s}}+\vec{V}_{\Gamma_{i}}
$$

The $\dot{\Gamma}$ term accounts for the blade's own near-wake shed vorticity, and $\vec{V}_{\Gamma_{i}}$ term accounts for the bound vortices of the other $N_{b}-1$ blades. The near-wake shed vorticity of the other blades is ignored. $\vec{L}$ is calculated directly from $\Gamma$

$$
\vec{L}=\rho \Gamma\left(\vec{V}_{c / 4} \times-\hat{z}\right)+\rho c \dot{\Gamma}\left(\vec{V}_{c / 4} \times-\hat{z}\right) / V_{c / 4},
$$

and a corresponding $c_{l}$ is found

$$
c_{l}=\frac{2\left(\Gamma+c \dot{\Gamma} / V_{c / 4}\right)}{c V_{c / 4}}
$$

Note that during iterations, the $\dot{\Gamma}$ used here is the derivative of the guess $\Gamma$. 2D airfoil section data are used to find $c_{d}$ and $c_{m}$ corresponding to the $c_{l}$ (see below). The drag and moment are calculated

$$
\begin{aligned}
\vec{D} & =\frac{1}{2} \rho \vec{V}_{c / 4}^{2} c c_{d}, \\
\vec{M} & =\frac{1}{2} \rho\left(\vec{V}_{c / 4}^{2} \times \hat{\eta}\right) c^{2} c_{m} .
\end{aligned}
$$

Note that the moment is positive nose down, so positive moment reinforces the motion.

\section{F. Drag and Moment Coefficient Model}

For each $\theta$, the associated $c_{l}$ is mapped to a $c_{d}$ and $c_{m}$ using blade profile characteristics ${ }^{6}$, augmented by the stall model. For $c_{l \min }<c_{l}<c_{l \max }$, the polar file data are interpolated using a $10^{\text {th }}$ order polynomial, thereby obtaining $c_{d}$ and $c_{m}$. The stall behavior implies a change in the $c_{d}$ curve for $c_{l}$ outside this range. The $c_{d}\left(c_{l}\right)$ imported from the polar files is augmented by a $c_{d_{\text {stall }}}$ :

$$
c_{d}=\left\{\begin{array}{cl}
c_{d}\left(c_{l}\right), & c_{l \min }<c_{l}<c_{l \max } \\
c_{d}\left(c_{l \max }\right)+\left.\frac{d c_{d}}{d c_{l}}\right|_{c_{\text {max }}}\left(c_{l}-c_{l \max }\right), & c_{l}>c_{l \text { max }} \\
c_{d}\left(c_{l \min }\right)+\left.\frac{d c_{d}}{d c_{l}}\right|_{c_{l \min }}\left(c_{l}-c_{l \min }\right), & c_{l}<c_{l \min }
\end{array} .\right.
$$

This defines how the drag polar should be extended through stall, which is a simple linear function $c_{d}\left(c_{l}\right)$, as shown in Fig. 4.

Note that, during iterations, the $c_{l}$ used here is calculated from the input $\Gamma$. Since the polynomials are quite invalid beyond the input data range, any $c_{l}$ outside the range of the polar file will give $c_{m}=0$. 


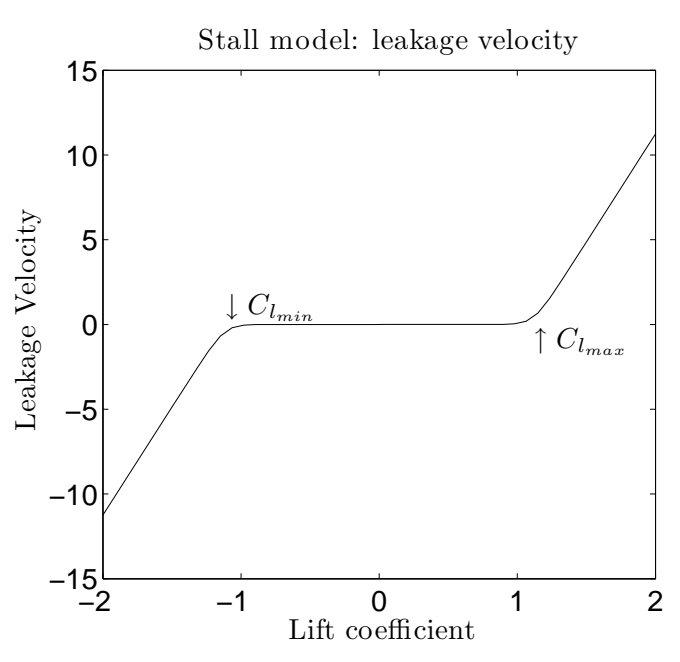

Figure 3. Control point leakage velocity for stall model.

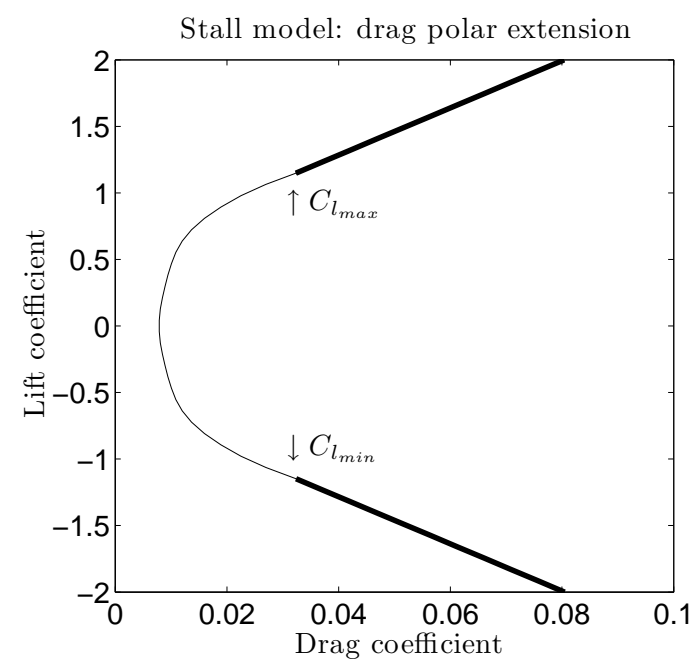

Figure 4. 2D profile drag polar

\section{G. Thrust and Power Coefficients}

The thrust, net moment about the origin, and power are calculated for one foil from the force, with positive power corresponding to the power extracted from the air,

$$
T=(\vec{L}+\vec{D}) \cdot \hat{x}, \quad P=\vec{M}_{\text {tot }} \cdot \vec{\Omega}=\left(\vec{M}+\vec{r}_{c / 4} \times(\vec{L}+\vec{D})\right) \cdot \vec{\Omega} .
$$

The thrust and power are averaged over the cycle (using the Midpoint rule for the integration), and the thrust and power coefficients for the entire turbine are then calculated

$$
C_{T}=\frac{\bar{T} N_{b}}{\frac{1}{2} \rho V_{\infty}^{2} A}, \quad C_{P}=\frac{\bar{P} N_{b}}{\frac{1}{2} \rho V_{\infty}^{3} A} .
$$

The thrust is normalized by the freestream dynamic pressure and turbine area, and the power extracted is normalized by the total power available in the external flow through the turbine region of area $A$.

\section{Solution Method}

All quantities are expressed in terms of $\theta$ and are implicitly assumed periodic. We discretize $\theta$ into $m$ points* $\theta_{i}=2 \pi i / m, i=0 . . m-1$. The unknowns that we solve for are then $\Gamma_{i}=\Gamma\left(\theta_{i}\right)$ and the averaged quantity $C_{T}$. Assuming that $\Gamma$ is sufficiently smooth, a spectrally accurate finite differentiation matrix ${ }^{7}$ is used to calculate $\dot{\Gamma}$ from $\Gamma$,

$$
\dot{\Gamma}_{j} \approx \sum_{i=-\frac{m}{2}+1}^{\frac{m}{2}-1} d_{i} \Gamma_{j+i}, \quad d_{i}=\left\{\begin{array}{cc}
\frac{1}{2}(-1)^{i+1} \cot \left(\frac{\pi i}{m}\right), & m \neq 0 \\
0, & m=0
\end{array}\right.
$$

Initial guesses to $\Gamma$ and $C_{T}$ are supplied by a simpler model (no stall or $\dot{\Gamma}$, which only requires iterations on $C_{T}$ ), or assumed zero. Newton iterations to drive point-wise residuals of Eqs. (2) and (5) to zero. The residuals are

$$
\begin{aligned}
& R_{\Gamma}=\left(-\frac{\vec{n}}{\pi} \Gamma+\vec{V}_{\mathrm{cp}}+\frac{-b}{\vec{V}_{\mathrm{cp}} \cdot \hat{\mathrm{s}}} \dot{\Gamma} \vec{n}\right) \cdot \vec{n}-C(\Gamma), \\
& R_{C_{T}}=C_{T}-\frac{\bar{T} N_{b}}{\frac{1}{2} \rho V_{\infty}^{2} A} .
\end{aligned}
$$

${ }^{*} m$ is taken as an integer multiple of the number of blades, we use $m=16$ for the results below.

$$
6 \text { of } 9
$$


The residuals are checked for convergence (using a predefined tolerance). A Jacobian matrix is constructed using analyitcal derivatives of the terms in the residuals**,

$$
J=\left[\begin{array}{cc}
\partial R_{\Gamma} / \partial \Gamma & \partial R_{\Gamma} / \partial C_{T} \\
\partial R_{C_{T}} / \partial \Gamma & \partial R_{C_{T}} / \partial C_{T}
\end{array}\right] .
$$

And finally the solutions are updated:

$$
\left[\begin{array}{c}
\Gamma \\
C_{T}
\end{array}\right]=\left[\begin{array}{c}
\Gamma \\
C_{T}
\end{array}\right]-J^{-1}\left[\begin{array}{c}
R_{\Gamma} \\
R_{C_{T}}
\end{array}\right]
$$

Because of the square root in the $\vec{V}_{\infty}^{\prime}$ equation, a $C_{T}>1$ will result in imaginary coefficients. The actual Newton step is scaled down in this case (under-relaxed Newton). This generally occurs with high solidity and high TSR, since this can result in $C_{T}$ very close to 1 .

\section{Optimization Results}

The model was wrapped in an optimization loop in order to study the isolated affects of TSR and $\alpha_{0}(\theta)$ on efficiency $\left(C_{P}\right)$. The pitch variation is assumed sinusoidal (one-per-cycle variation) so

$$
\alpha_{0}=A_{0}+A_{1} \sin (\theta+\phi),
$$

where $A_{0}$ is the pitch offset, $A_{1}$ is the oscillation amplitude, and $\phi$ is the phase. In addition, the affect of 'dirty blades' was simulated with increasing factors multiplying $c_{d}$. In all of the cases below, we report $C_{P}$ versus TSR, with varying $\alpha_{0}$ parameters or $c_{d}$ factors. All designs have three blades with NACA0012 airfoils, a reference $\operatorname{Re}=$ $3 \times 10^{5}$, a streamtube deceleration factor* of $\ell=6 R$, and optimized $\sigma$. In addition, the baseline case is optimized for all parameters (with the $c_{d}$ factor $K_{c_{d}}=1$ ), and each figure represents sensitivity of the baseline to variations in a single parameter. The baseline (optimized) case has $\sigma=0.29$, TSR $=2.17$, and a pitch modulation function $\alpha_{0}=7.6+6.2 \sin (\theta+21.5)$ degrees, which yields $C_{P}=0.534$.

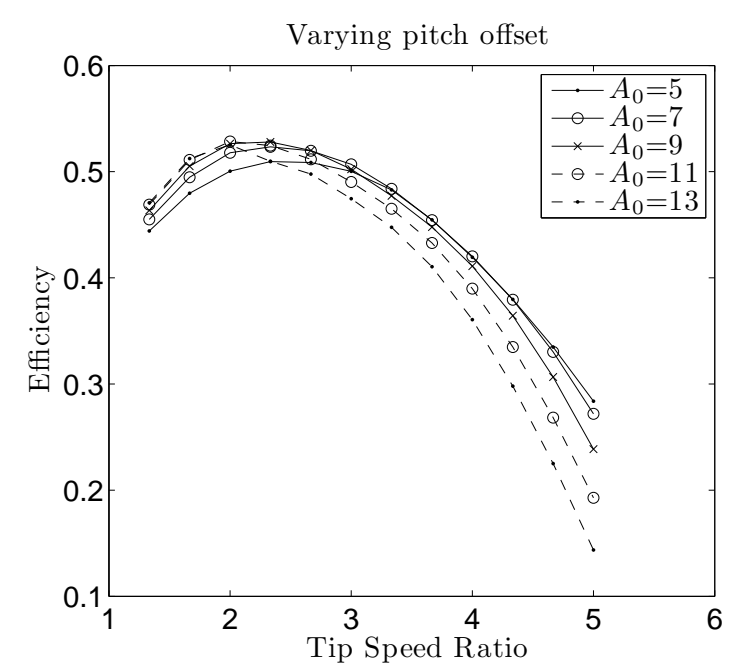

Figure 5. Efficiency vs tip speed ratio and pitch offset $A_{0}$

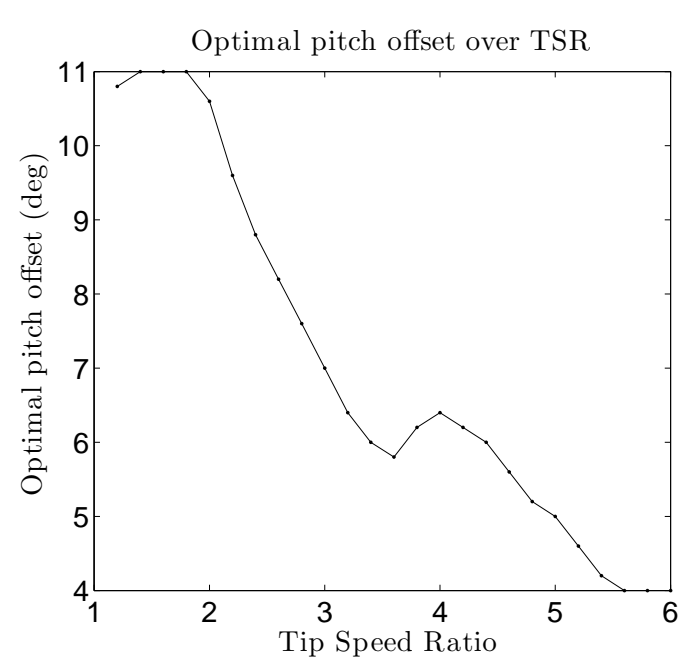

Figure 6. Variation of optimal pitch offset $A_{0}$ with tip speed ratio

First, we vary the pitch offset $A_{0}$, while keeping the sinusoidal component constant and $K_{c_{d}}=1$. Fig. 5 shows that there is an optimal $A_{0}$, since the peak efficiency increases and then decreases with $A_{0}$. In addition, as $A_{0}$ increases, the dependence on TSR increases. This is essentially the increased sensitivity of operating the turbine closer to stall conditions. In addition, the peak efficiency shifts slightly toward lower TSR, where the pitch variation takes advantage of the larger variation in blade-relative velocities. These trends can be seen in the variation of the optimal $A_{0}$ with TSR, as shown in Fig. 6.

\footnotetext{
** The effects of one main vortex on another are small and not included in calculating the derivatives due to complexity. This does not appear to affect the convergence of the residuals.

${ }^{*}$ Chosen to agree with experimental results: D. W. Erickson, J. J. Wallace and J. Peraire (In preparation)
} 
In Fig. 7, the pitch oscillation amplitude $A_{1}$ is varied. Larger $A_{1}$ tends to decrease the maximum efficiency point (excessive stall) and shift it toward smaller TSR. Again, the dependence on TSR is stronger for higher $A_{1}$ due to operating closer to the stall limits.
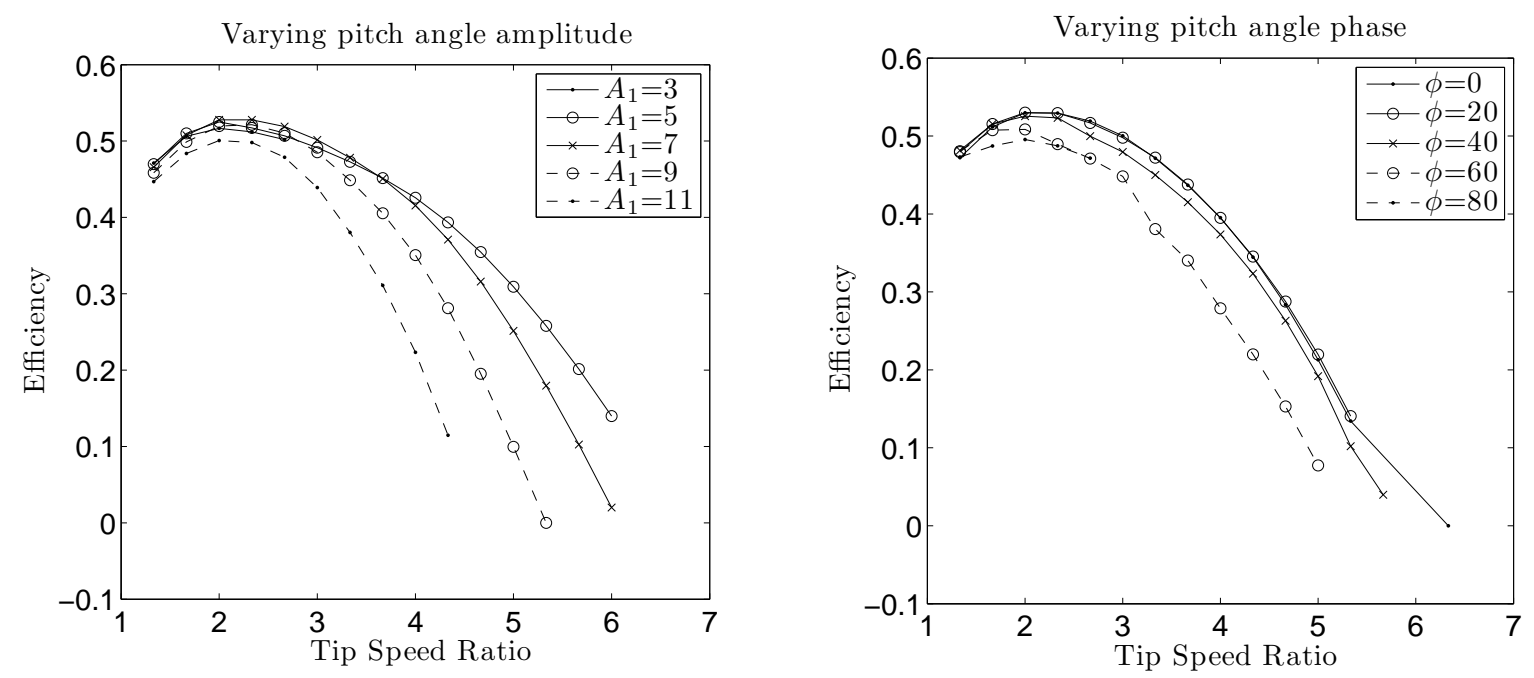

Figure 7. Efficiency vs tip speed ratio and pitch modulation ampli- Figure 8. Efficiency vs tip speed ratio and pitch modulation phase tude $A_{1}$ angle $\phi$

In Fig. 8, the phase $\phi$ is varied. As can be seen, the efficiency is somewhat sensitive to the phase angle. Phase lead reduces the peak efficiency and reduces off-design performance (i.e. efficiency at higher TSR). Interestingly, this effect is very weak at low TSR.

Finally, variation of the $c_{d}$ factor is shown in Fig. 9. Clearly, larger $c_{d}$ reduces efficiency. In addition, larger $K_{c_{d}}$ shifts the optimal TSR downward, since operating at a higher TSR is more sensitive to drag.

Note that in all of the above cases, $\sigma=c / R$ (solitidy) is optimized. This is done because there is a relationship between $\sigma$ and TSR in the optimal case, specifically $\sigma$ TSR $\approx$ constant. Since TSR is a ratio of velocities and $\sigma$ is a ratio of length scales, $\sigma$ TSR is a ratio of time scales between the turbine $(\Omega)$ and the freestream $\left(c / \vec{V}_{\infty}\right)$. In an optimized design, the velocity, length, and time scales are all matched. Fig. 10 shows this trend for the baseline case (only varying TSR).

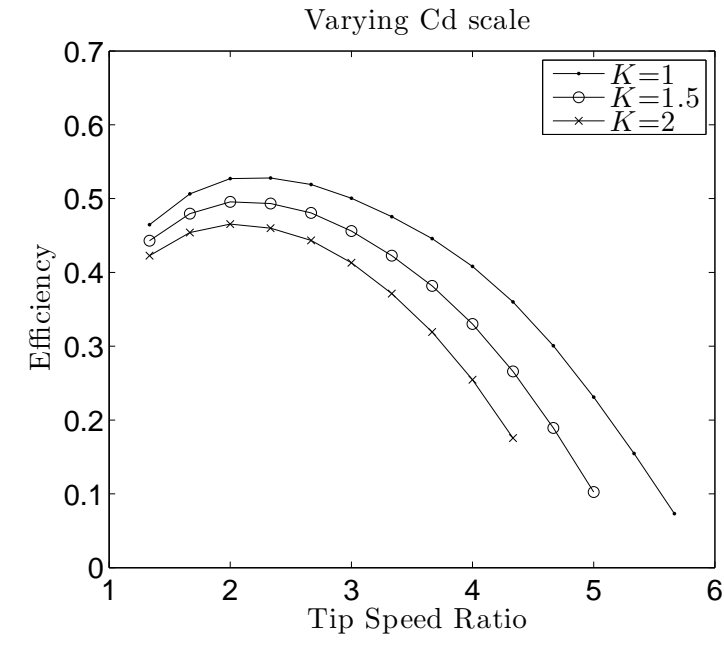

Figure 9. Efficiency vs tip speed ratio and drag coefficient factor $K_{c_{d}}$

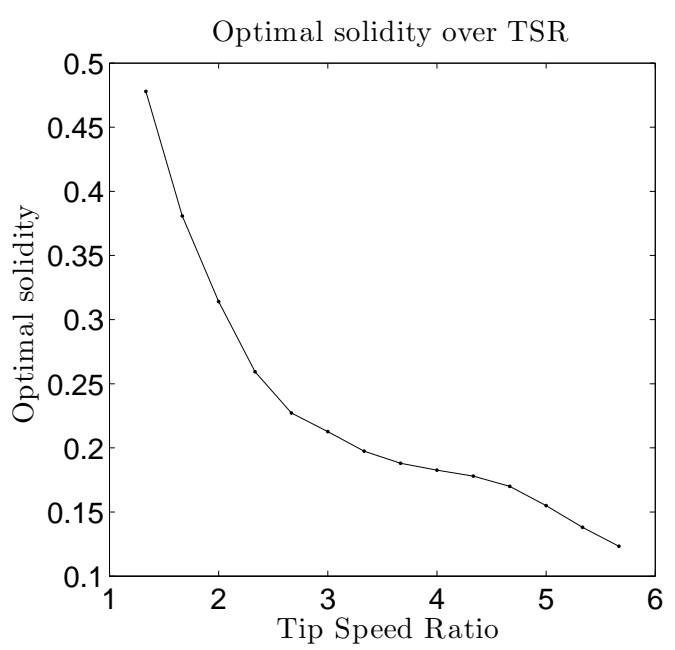

Figure 10. Variation of optimal solitidy $\sigma$ with tip speed ratio 


\section{Future Work}

Further improvements to the model would include accounting for dynamic stall and Reynolds number variation over the angle $\theta$. The model converges well for moderate to low $\sigma$, moderate to high TSR, and moderate $\alpha_{0}$. Dynamic stall modeling may give better accuracy at high $\sigma$ and low TSR, but the model has been shown to be a good approximation to a high fidelity 2D Navier-Stokes solution with high solidity and low tip speed ratio. The high fidelity solution has vortices shedding at the airfoil leading edge (dynamic stall), which produces fluctuations in the resulting $\vec{F}_{\text {tot }}(\theta)$ and $\vec{M}_{\text {tot }}(\theta)$. The low order model predicts forces and moments that are close to the high fidelity results in regions without dynamic stall, and the overall $C_{P}$ is similar. Reynolds number variation over $\theta$ would have to be implemented as an empirical $c_{d}$ scaling and would not change the model results much for moderate to high TSR.

This model is particularly easy to extend to various pitch control mechanisms. First of all, an optimal $\alpha_{0}(\theta)$ variation can be computed by wrapping the model in an optimization loop. This can be compared to forced one-percycle pitch variation, where $\alpha_{0}(\theta)=A_{0}+A_{1} \sin (\theta+\phi)$. One could also include passive pitch control mechanisms such as a spring or an aerodynamic spring. This is simply done by introducing new state variables $\alpha_{0}(\theta)$ and $\dot{\alpha}_{0}(\theta)$ and calculating the appropriate additional velocities and forces.

\section{References}

\footnotetext{
${ }^{1}$ Templin, R. J., "Aerodynamic Performance Theory for the NRC Vertical-Axis Wind Turbine," NASA STI/Recon Technical Report N, Vol. 76, June 1974, pp. 16618-+.

${ }^{2}$ Strickland, J. H., "A Performance Prediction Model for the Darrieus Turbine," International Symposium on Wind Energy Systems., British Hydrodynamics Research Association, Cranfield, Beds., England, 1977, pp. 3-+.

${ }^{3}$ Paraschivoiu, I., "Double-Multiple Streamtube Model for Studying Vertical-Axis Wind Turbines," Journal of Propulsion Power, Vol. 4, Aug. 1988, pp. 370-377.

${ }^{4}$ Strickland, J. H., Webster, B. T., and Nguyen, T., "Vortex Model of the Darrieus Turbine: An Analytical and Experimental Study," NASA STI/Recon Technical Report N, Vol. 80, Feb. 1980, pp. 25887-+.

${ }^{5}$ Drela, M., "Integrated Simulation Model for Preliminary Aerodynamic, Structural, and Control-Law Design of Aircraft," AIAA/ASME/ASCE/AHS/ASC Structures, Structural Dynamics, and Materials Conference and Exhibit, Vol. 3, AIAA, Washington, DC, 1999.

${ }^{6}$ Drela, M., "XFOIL: An Analysis and Design System for Low Reynolds Number Airfoils," Conference on Low Reynolds Number Airfoil Aerodynamics, University of Notre Dame, June 1989.

${ }^{7}$ Gopinath, A. K. and Jameson, A., "Time Spectral Method for Periodic Unsteady Computations over Two- and Three- Dimensional Bodies," 43rd AIAA Aerospace Sciences Meeting and Exhibit, AIAA, Washington, DC, Jan. 2005.
} 